Cerebrovasc Dis 2006;22:209-213

DOI: $10.1159 / 000093811$

\section{Traumatic Dissection of the Vertebral Artery - Value of Sonographic Diagnostics}

Eva Bartels ${ }^{a}$, Michael Knauth ${ }^{b}$, David Liebetanz ${ }^{a}$, Walter Paulus ${ }^{a}$

Departments of a Clinical Neurophysiology and

${ }^{\mathrm{b}}$ Neuroradiology, Georg-August University Göttingen,

Göttingen, Germany

\section{Introduction}

Dissection of a cervical artery supplying the brain is a major cause of stroke in young adults. It can occur spontaneously, in connection with an underlying disorder such as fibromuscular dysplasia, $\alpha_{1}$-antitrypsin deficiency, hypertension, migraine, atherosclerosis, arteritis or Marfan's disease [1, 2]. More often, it is related to

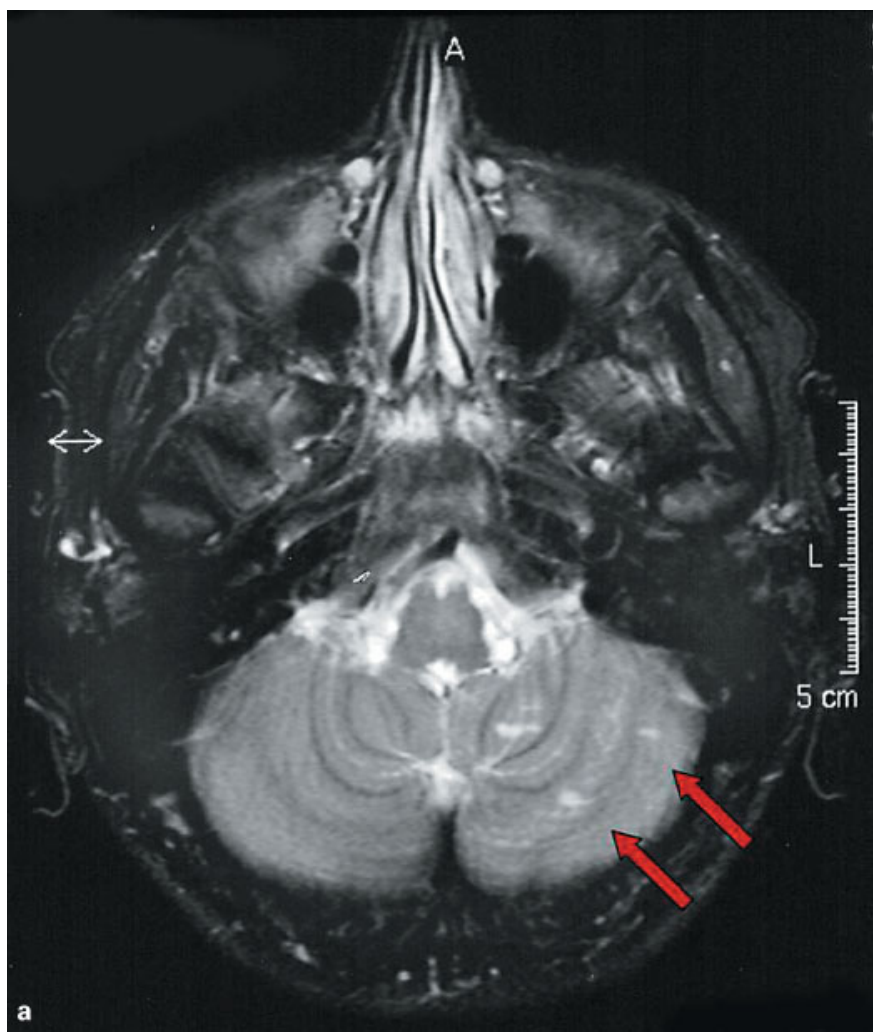

a neck trauma. In the case of a vertebral artery dissection, a vessel wall can be injured through abrupt or continuous neck movements, regardless of visible outward signs of neck trauma. The first clinical symptoms - occipital headache, neck pain and vertigo - can be associated with focal neurological deficits of varying severity, from transient ischemic attacks up to complete ischemia in the vertebrobasilar territory [3].

Dissection of the vertebral arteries is most accurately diagnosed with conventional digital subtraction angiography (DSA). In recent years, this procedure is being increasingly replaced by noninvasive techniques, such as magnetic resonance imaging (MRI), MR angiography and duplex ultrasonography [4-7]. We describe a traumatic dissection of the left vertebral artery in the distal V2 segment which was initially not recognized using extracranial duplex sonography - although the examination had been carried out with a view to this very possibility.

\section{Case Report}

A few days before admission, this 40-year-old white woman had already complained about recurring episodes of vertigo and nausea that sometimes lasted for up to $1 \mathrm{~h}$. In addition, the patient complained about occipital headache and recurring scotoma, similar to those scotomas she knew from migraine attacks, although these were much more prolonged. She had no personal or family history of thromboembolic disease, no genetic disorder and did not smoke

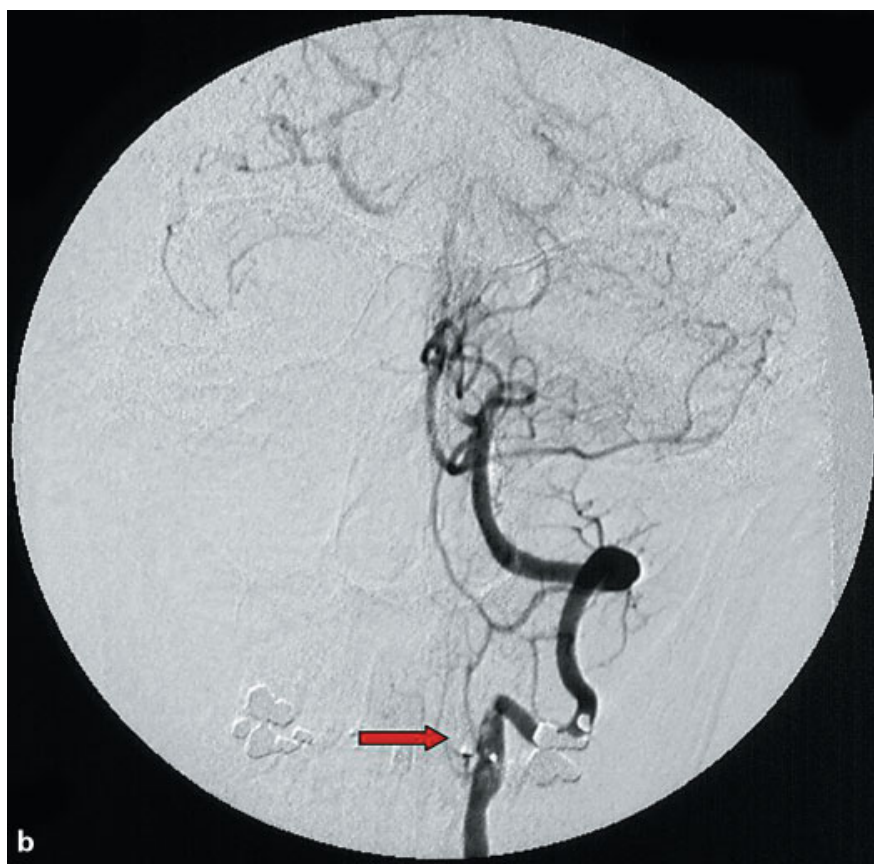

Fig. 1-3. Findings in a 40-year-old woman with a dissection of the left vertebral artery in a V2/V3 segment after a moderate head and neck trauma, i.e. a blow on the temple from the pole of a garden umbrella, 2 weeks before the onset of the clinical symptoms.

Fig. 1. MRI and DSA findings. a MRI image showing multiple lesions of high signal intensity in the left cerebellar hemisphere (arrows). b DSA findings of the left vertebral artery show a short narrowing at the atlas loop and a proximal enlargement of the vessel lumen at about the level of the C3 vertebra (arrow). 


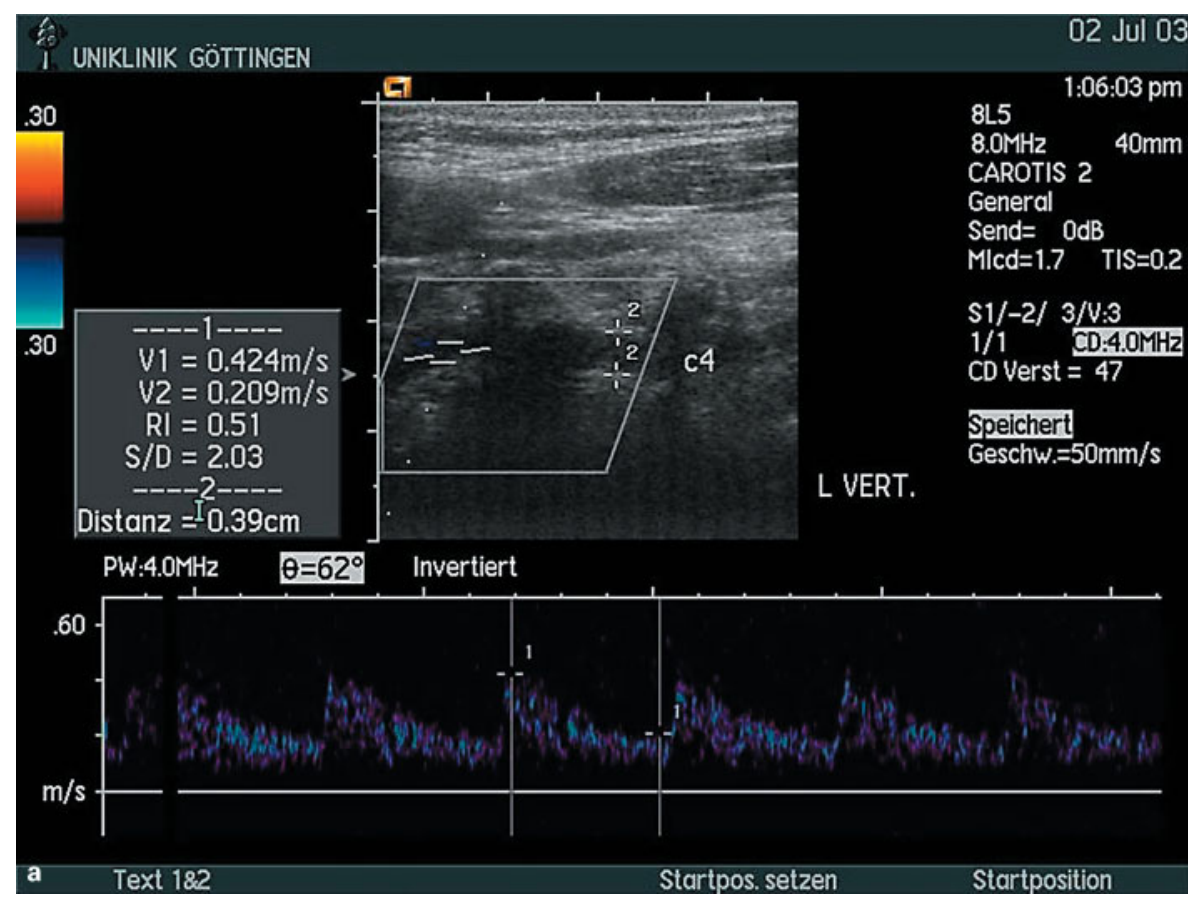

Fig. 2. Color-coded duplex ultrasonography findings 2.5 weeks after the trauma. a View of the midcervical course of the left vertebral artery (between the V4 and V 3 cervical vertebrae) showing a normal Doppler pulse curve and a normal-appearing vessel lumen with a diameter of $3.9 \mathrm{~mm}$. b In an examination focusing on the segment at the level of the C3 vertebra, a distal enlargement of the vessel lumen can be recognized. (Diameter of the vertebral artery proximally from the $\mathrm{C} 3$ vertebra is $3.9 \mathrm{~mm}$, distally from the $\mathrm{C} 3$ vertebra $5.6 \mathrm{~mm}$.) The delineation of the vessel wall is irregular - a typical finding in the case of a dissection. $\mathbf{c}, \mathbf{d}$ View of an intimal flap (arrows), which can be seen as a structure independent of the vessel wall, pulsating in synchronization with the cardiac cycle in the lumen of the vertebral artery at the point of entry of the vessel into the transverse foramen of the third vertebra. Video sequences No. 1 (c) and No. 2 (d).

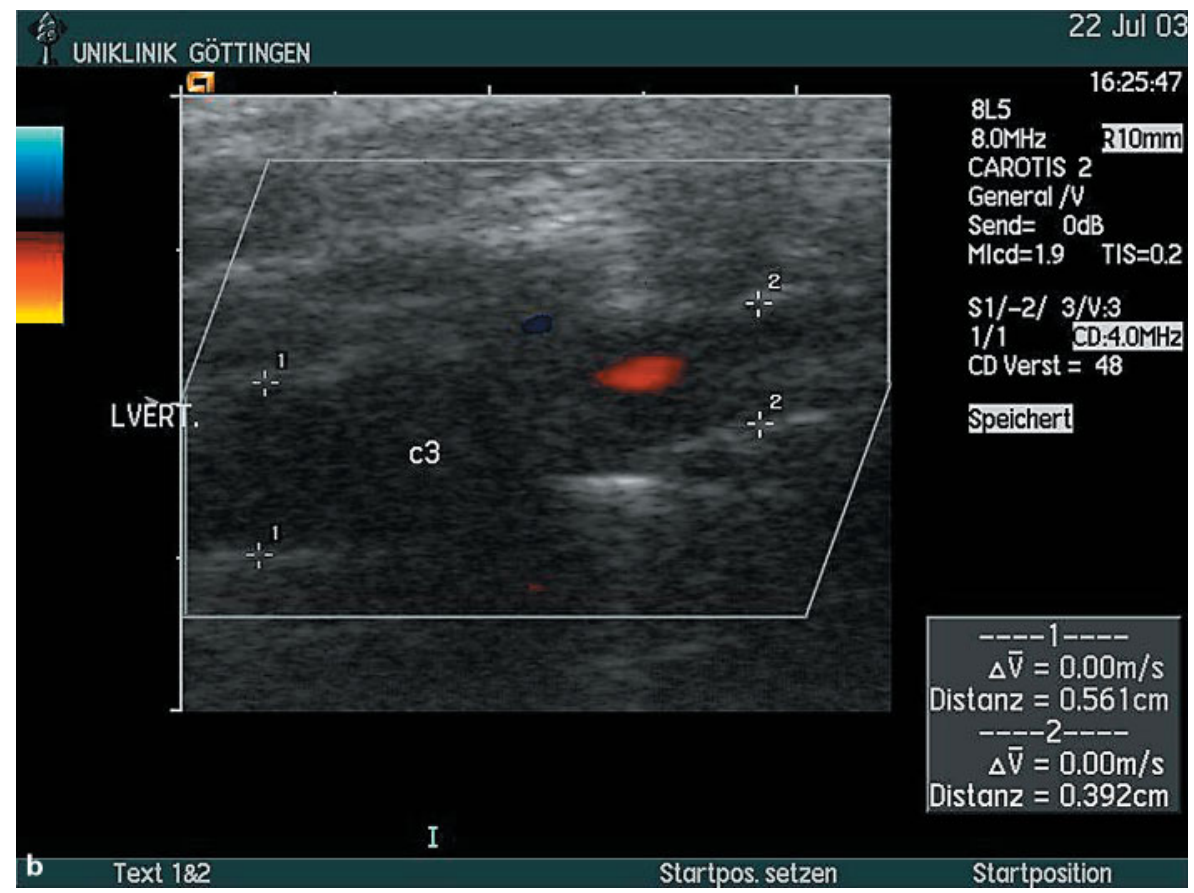

or use oral contraceptives. About 10 days before admission, the patient was struck on the left temple by the pole of a garden umbrella, which resulted in a hematoma.

On admission, the initial clinical examination revealed a normal neurological state except for a discrete horizontal spontaneous nystagmus beating to the left side. From the start, a traumatic dis- section of the vertebral artery was an early consideration. However, the initial sonographic examination showed no pathological findings in the extracranial course of the vertebral arteries. The MR angiography was also normal. The MRI scan showed multiple small lesions and a leptomeningeal enhancement in the left cerebellar hemisphere (fig. 1a). 

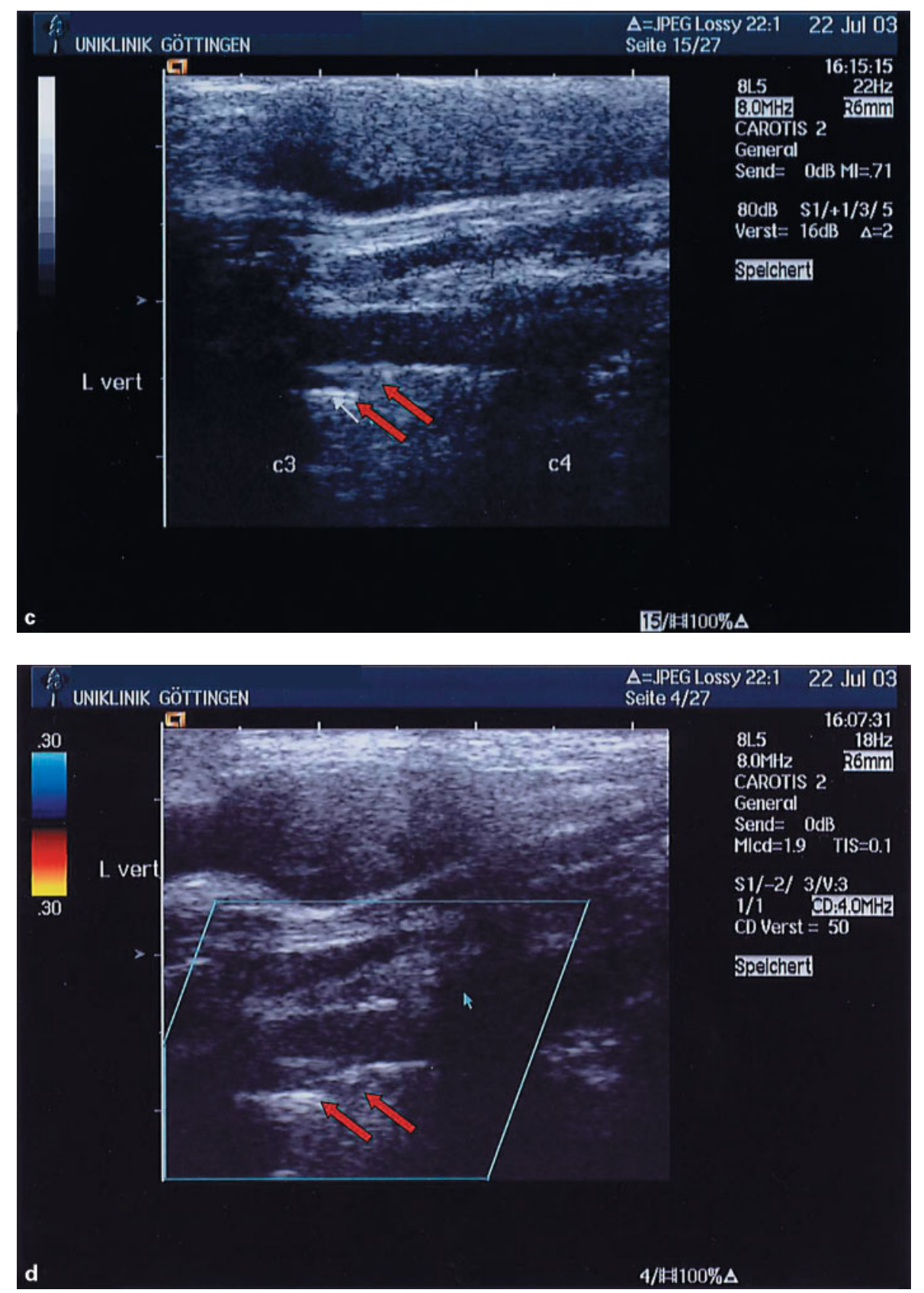

Two days after admission, the patient suddenly complained of an increase in rotational vertigo and nausea. In consequence, a cardiovascular investigation, follow-up continuous-wave Doppler sonography, transcranial Doppler sonography and color-coded duplex ultrasonography were carried out, but all showed normal findings. No evidence was found for the suspected dissection in the extracranial course of the left vertebral artery. Because of the progressing cerebellar symptoms, a selective DSA of the cervical and cerebral arteries was performed on the same day.
This revealed a short stenosis of the vertebral artery at the transition of the V2 to the V3 segment. Several irregularities and an enlargement of the vessel wall were seen prior to stenosis (fig. 1b). In view of these angiographic findings, a further color-coded duplex ultrasonography of the extra- and intracranial vertebral arteries was performed. A special examination was made of the segment between the $\mathrm{C} 3 / \mathrm{C} 2$ cervical vertebrae, which showed the vessel wall to have an irregular surface with a moving intimal flap. This appeared as a structure independent of the vessel wall that moved in synchronization with the cardiac cycle (fig. 2). 
Fig. 3. Follow-up 6 weeks after the examination shown in figure 2. Examination in the cranial V2 segment of the left vertebral artery (in the same sonographic plane as displayed in figure $2 b$ ). The enlargement of the vessel in the cranial area is less prominent. (Diameter of the vertebral artery proximally from the $\mathrm{C} 3$ vertebra is $3.8 \mathrm{~mm}$, distally from the $\mathrm{C} 3$ vertebra $4.6 \mathrm{~mm}$.) The vessel wall is better delineated (arrow); however, in the segment located distally from the C3 vertebra, an irregular surface can still be recognized. The pulsating intimal flap is no longer visible. Video sequence No. 3.

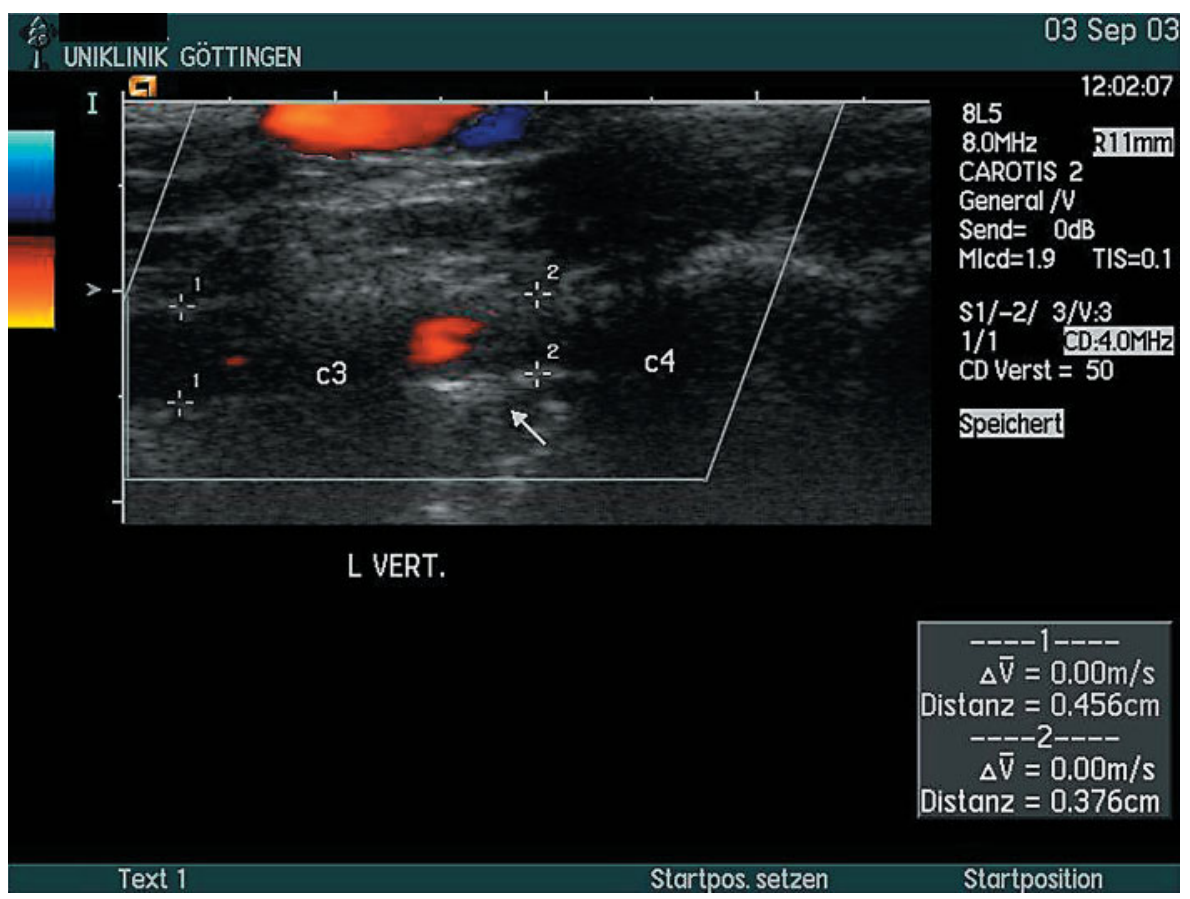

The patient was then treated with anticoagulants for 6 months. Neurological symptoms disappeared within the first 3 days and remained absent. The ultrasonographic follow-up during the subsequent 6 months showed healing of the intravascular lesion (fig. 3)

\section{Discussion}

With the progress of high-resolution ultrasonographic techniques, it is increasingly possible to noninvasively diagnose dissection of an extracranial vertebral artery $[8,9]$. Duplex ultrasonography can reveal conditions that vary from irregular thickening of the vessel wall without hemodynamic alteration to major structural lesions [10].

In our study of 24 patients with 28 extracranial vertebral artery dissections, the most frequent dissection site was the entrance of the vertebral artery in the transverse foramen of the C6 vertebra - in the midcervical course, between the V1 and V2 segments [11]. In this region, the artery is possibly exposed to the greatest mechanical injury. Particularly in this region, the vertebral artery is also easily accessible for color-coded duplex ultrasonography.

The second most frequent site is the origin (segment V0/proximal V1 segment) of the vertebral artery. Additionally, alterations in the vessel wall can be imaged directly [12].

In contrast, a dissection located in the V 3 segment, at the atlas loop or at the craniocervical transition point, cannot be visualized directly on the screen. The diagnosis of a stenosis or occlusion in this region is based only on indirect signs recorded in the midcervical course, such as a high resistance flow pattern indicating a distally obstructed flow [10].

In our patient, a dissection was localized in this very cranial V2 segment and in the V3 segment, and was initially overlooked in the sonographic study because no signs of a distal flow obstruction were present in the well-visible midcervical course. This can be explained by a dilation of the vessel lumen at the level of the C3 vertebra as displayed with angiography (fig. 1b). The advantage of duplex sonography only became apparent with the control examination, which consequently focused on the area detected angiographically. Here, a movement, namely an intimal flap, could be visualized reproducibly in the vessel lumen in real time (fig. 2c, d, video sequences No. 1 and 2). Follow-ups during the subsequent 6 months showed healing of the intima tear which also resulted in improvement in the clinical status (fig. 3, video sequence No. 3).

In conclusion, if a dissection is located in a segment which cannot be displayed by duplex ultrasonography, conventional angiography, MR angiography or MRI with fat suppression technique can find and narrow the area of diagnostic focus. The advantage of duplex sonography then lies in its ability to produce, in real-time and in subsequent follow-ups, an exact representation of the alterations localized via angiography.

\section{References}

1 Caplan LR, Tettenborn B: Vertebrobasilar occlusive disease: review of selected aspects. 1. Spontaneous dissection of extracranial and intracranial posterior circulation arteries. Cerebrovasc Dis 1992;2:256-265.

2 Konrad C, Nabavi DG, Junker R, Dziewas R, Henningsen H, Stogbauer F: Spontaneous internal carotid artery dissection and alpha-1-antitrypsin deficiency. Acta Neurol Scand 2003;107:233-236.

3 Caplan LR, Amarenco P, Rosengart A, Lafranchise EF, Teal PA, Belkin M, DeWitt LD, Pessin MS: Embolism from vertebral artery origin occlusive disease. Neurology 192;42:1505-1512. 
4 Sturzenegger M, Mattle HP, Rivoir AS, Baumgartner RW: Ultrasound findings in carotid artery dissection: analysis of 43 patients. Neurology 1995;45:691-698

5 Zuber J, Meary E, Meder JF, Mas JL: Magnetic resonance imaging and dynamic CT scan in cervical artery dissections. Stroke 1994;25:576581.

6 Bui LN, Brant-Zawadzki M, Verghese P, Gillan G: Magnetic resonance angiography of cervicocranial dissection. Stroke 1993;24:126-131.

7 Steinke W, Rautenberg W, Schwartz A, Hennerici M: Noninvasive monitoring of internal carotid artery dissection. Stroke 1994;25:998-1005.

8 Bartels E, Flügel KA: Evaluation of extracranial vertebral artery dissection with duplex color flow imaging. Stroke 1996;27:290-295.

9 Touboul PJ, Mas JL, Bousser MG, Laplane D: Duplex scanning in extracranial vertebral artery dissection. Stroke 1987;18:116-121.

10 Bartels E: Dissection of the vertebral arteries; in Bartels E (ed): ColorCoded Duplex Ultrasonography of the Cerebral Vessels/Atlas and Manual. Stuttgart, Schattauer, 1999, pp 125-127.

11 Bartels E: Dissection of the extracranial vertebral artery: clinical findings and early noninvasive diagnosis in 24 patients. J Neuroimag 2006;16: 24-33.

12 Saito K, Kimura K, Nagatsuka K, Nagano K, Minematsu K, Ueno S, Naritomi H: Vertebral artery occlusion in duplex color-coded ultrasonography. Stroke 2004;35:1068.

Eva Bartels, MD, Department of Clinical Neurophysiology Georg-August University Göttingen, Robert-Koch-Strasse 40 DE-37075 Göttingen (Germany)

Tel. +4989297 216, Fax +49892421 7799

E-Mail Bartels.Eva@t-online.de
Cerebrovasc Dis 2006;22:213-214

DOI: $10.1159 / 000093812$

\section{Fatal Embolic Myocardial Infarction after Systemic Thrombolysis for Stroke}

\author{
Wassilios Meissner ${ }^{a}$, Thomas Lempert ${ }^{a}$, \\ Sabine Saeuberlich-Knigge ${ }^{b}$, Wolfgang Bocksch ${ }^{c}$, \\ Ulrich-Frank Pape ${ }^{d}$
}

Departments of a Neurology, ${ }^{b}$ Nephrology and Intensive Care, ${ }^{\mathrm{C} C a r d i o l o g y}$, and ${ }^{\mathrm{d}}$ Hepatology, Gastroenterology, Endocrinology and Metabolism, Charité Campus VirchowKlinikum, Humboldt University Berlin, Berlin, Germany

\section{Introduction}

Systemic intravenous thrombolysis in stroke patients improves long-term clinical outcome when administered within $3 \mathrm{~h}$ following onset of the neurological deficit [1]. Therefore, systemic intravenous thrombolysis has been established as a first-line therapeutic strategy in patients admitted for stroke meeting specific criteria with cerebral hemorrhage being the main complication [1]. Since then, thrombolyis has proven to be cost-effective [2, 3], but only less than $4 \%$ of stroke patients currently benefit from thrombolytic treatment [4].

Although cardioembolism is the cause of $20-40 \%$ of all strokes $[5,6]$ and $26 \%$ of patients with an acute ischemic stroke or a transient ischemic attack have an intracardiac thrombus [7,
8], the risk for systemic embolism from a pre-existing intracardiac thrombus in stroke patients is unknown [9]. Here we report a patient who received systemic thrombolysis for stroke and who developed a finally fatal embolic MI from a presumably pre-existing atrial thrombus $2 \mathrm{~h}$ after initiation of systemic thrombolysis.

\section{Case Report}

A 62-year-old woman with a medical history of atrial fibrillation, arterial hypertension, diabetes mellitus and obesity was admitted to the emergency room for acute left brachiofacial hemiparesis and forced gaze deviation to the right. Initial laboratory evaluation showed unremarkable cardiac enzymes and coagulation parameters. A brain CT was normal except for a right hyperdense middle cerebral artery (MCA) pointing to occlusion of the vessel. The electrocardiogram (ECG) showed atrial fibrillation. Systemic thrombolysis with intravenous rt-PA (6.3 mg bolus, $56.7 \mathrm{mg}$ for $1 \mathrm{~h}$ ) was administered according to the guidelines of the NINDS rt-PA Stroke Study Group [1] starting two hours after the onset of the neurological symptoms. Two hours after rt-PA treatment the patient developed cardiogenic shock, required catecholamine therapy and was intubated for mechanical ventilation. ST segment elevations characteristic of anterior myocardial infarction appeared in ECG leads V2-V6 with reciprocal ST segment depression in the leads II, III and $\mathrm{aVF}$. At this time creatine kinase (CK), creatine kinase-MB (CK$\mathrm{MB})$, and troponin $\mathrm{T}(\mathrm{TnT})$ levels were within normal limits. Immediate coronary angiography showed a complete occlusion of the left anterior descending coronary artery (LAD) and an abrupt occlusion of a small posterolateral branch of the left circumflex coronary artery. The residual coronary arterial tree was smooth without any lumen irregularities. Rescue percutaneous transluminal coronary angioplasty (PTCA) of the LAD was performed with a $25 \%$ residual diameter stenosis and a delayed distal run off of the contrast dye (grade II flow according to the Thrombolysis In Myocardial Infarction (TIMI) classification for coronary reperfusion) suggesting microcirculatory thromboembolism. Transesophageal echocardiography showed decreased left ventricular ejection fraction due to extended anterior, anteroseptal and apical akinesia. The left atrium was enlarged, while spontaneous echocontrast was documented and a large inhomogeneous mass $(1.8 \times 2.3 \mathrm{~cm})$ consistent with a thrombus adjacent to the lateral atrial wall was detected. Twelve hours after PTCA CK rose to 3,040 U/1 (CK-MB $196 \mathrm{U} / 1$ ) and TnT levels increased to $17.98 \mu \mathrm{g} / \mathrm{l}$. The patient showed hemodynamic deterioration and died within $24 \mathrm{~h}$ in global cardiac failure refractory to intensive medical therapy.

\section{Discussion}

This patient had a fatal embolic MI after systemic thrombolysis for stroke. Key clinical findings were a past medical history with atrial fibrillation, a previously unknown pre-existing thrombus in the left atrium, a right hyperdense MCA as a feature of large artery occlusion leading to acute ischemic stroke, and an abrupt occlusion of the LAD without atheromatous alterations on coronary angiography as the cause for acute MI. These changes strongly suggest a thromboembolic etiology of both the stroke and the MI most likely originating from the left atrial thrombus. Both thromboembolic events may be explained by spontaneous fragmentation of the inhomogeneous mass in the left atrium, but con- 